\title{
RED DE SENSORES INALÁMBRICOS PARA EL MONITOREO DE VARIABLES AGROECOLÓGICAS EN CULTIVOS BAJO INVERNADERO
}

\author{
NETWORK OF WIRELESS SENSORS FOR MONITORING \\ AGROECOLOGICAL VARIABLES IN GREENHOUSE CROPS
}

\begin{abstract}
${ }^{1}$ Duvan David Barbosa Pira , ${ }^{2} J u a n$ Sebastián Sanabria Rodríguez, ${ }^{3}$ Hassler Camilo Bueno Mesa, ${ }^{4}$ Daniel Vega Castro, ${ }^{5}$ Edgar Aguirre Buenaventura.
\end{abstract}

Corporación Universitaria Minuto de Dios, Bogotá, Colombia.

\section{RESUMEN}

En la actualidad, el desarrollo de la agrónica para la automatización de procesos del sector agrícola y pecuario, está marcando una fuerte tendencia en la toma de decisiones con datos de variables agroecológicas, enfrentándose a problemas tecnológicos como la toma de datos de cultivos a diferentes escalas y bajo costo económico de implementación, por lo que esta investigación se enfocó en diseñar e implementar una red de monitoreo de variables agroecológicas en ambientes controlados, con la finalidad de encontrar y probar tecnologías que permitan mejorar la competitividad del sector primario de la economía del país. Lo anterior, con el monitoreo de variables a través de dispositivos electrónicos de medición y trasmisión de datos en tiempos reales a dispositivos móviles y ordenadores.

Palabras clave: agroníca, automatización, redes de sensores, monitoreo.

\section{Abstract}

Currently, the development of agronica for the automation of processes in the agricultural and livestock sector, is setting a strong trend in decision-making with data on agroecological variables, facing technological problems such as capture of crop data at different scales and low economic cost of implementation.

Keywords: agrónica, automation, monitoring, sensor networks.

\footnotetext{
${ }^{1}$ duvandavidbarbosa@hotmail.com, orcid.org//0000-0001-9508-3715

2 jsanabriarleuniminuto.edu.co, orcid.org//0000-0001-5341-547

3 cris-ha-ssahotmail.com, orcid.org//0000-0003-2321-0003

${ }^{4}$ agroecologiavega@gmail.com,orcid.org//0000-0001-7711-5437

${ }^{5}$ eaguirre@uniminuto.edu, orcid.org//0000-0001-7711-5437
} 


\section{INTRODUCCIÓN}

El diseño, desarrollo e implementación de sensores para la medición de variables agroecológicas (temperatura, humedad relativa, brillo solar, entre otras), bajo condiciones controladas, busca ofrecer insumos al sector agrícola que permitan además de monitorear el desarrollo fenológico de los cultivos, acciones asociadas a la automatización (Rodríguez, 2006; Muñoz \& Nuñez, 2012; Aguado da Costa, 2012; Carrillo \& Vázquez, 2008; Rodríguez et al., 2011) de procesos que repercuten de forma directa en calidad y producción de los alimentos obtenidos, reducción de costos de producción y procesamiento de la información, racionalización de las materias primas, agua y energía y además mejores índices HSEQ-calidad, salud, seguridad y ambiente.

En relación con lo anterior, Gorini (Alpi \& Tognoni, 1999) define un ambiente controlado como una estructura de madera o de hierro u otro material, cubierta por cristales y/o plástico, provista por lo general de calefacción que, a veces, está iluminada artificialmente y en donde se pueden cultivar hortalizas tempranas, flores y otro tipo de plantas. Autores como (Acuña Caita, 2009) informan del comienzo de los invernaderos en Colombia, y afirman que las primeras exportaciones son realizadas por la agroindustria de la floricultura en la década de los años 60.

Por otra parte, al hablar de invernaderos con sensores y automatización desde el contexto local, se puede decir que las primeras iniciativas a escala son gestadas bajo el marco del proyecto denominado "Fortalecimiento de la capacidad de investigación en ambientes controlados", desarrollado por la Corporación Colombiana de Investigación Agropecuaria, Corpoica y el Instituto Colombiano Agropecuario ICA. Lo anterior con recursos del Ministerio de Agricultura y Desarrollo Rural de Colombia. "Los invernaderos serán computarizados, de última generación y conectados a estaciones climáticas. En ellos se evaluará la adaptación de las especies bajo condiciones protegidas y se determinarán las condiciones óptimas para lograr altos niveles de productividad" (Portafolio, 2008).
Al entrar en contexto con la producción de biomasa ya sea representada en productos para el consumo directo (frutas, hortalizas y gramíneas) o indirecto (ornamentales de corte o de matera), se destacan variables que afectan de manera significativa la producción. Dentro de estos factores se destacan los cambios climáticos abruptos que afectan de forma directa el desarrollo y producción de los sistemas agrícolas, casos puntuales, los fenómenos conocidos con los nombres de El Niño, La Niña Oscilación del Sur - ENOS, los cuales son causa de la mayor señal de variabilidad climática en la franja tropical del Océano Pacífico, en la escala interanual. El Niño y su fase opuesta La Niña, son los componentes oceánicos del ENOS y corresponden, en términos generales, a la aparición, de tiempo en tiempo, de aguas superficiales relativamente más cálidas (El Niño) o más frías (La Niña), que lo normal, en el Pacífico tropical central y oriental, frente a las costas del norte de Perú, Ecuador y sur de Colombia. (Montealegre, 2007).

Así mismo, el tema asociado a las plagas, las cuales son definidas (Vega, 2011) como cualquier individuo capaz de generar alteraciones fisiológicas a los cultivos, generando dańos económicos para el productor. Dentro de las plagas más frecuentes se destacan artrópodos, ácaros y microorganismos como bacterias virus y hongos, entre otras.

Por lo anteriormente expresado, la pregunta de investigación hace referencia a ¡cómo monitorear diferentes variables agroecologías que permitan a través de los datos almacenados determinar el comportamiento de estas por medio de la estadística descriptiva?

La contribución de esta, consiste en el diseño e implementación de una red de monitoreo de variables agroecológicas en ambientes controlados (invernaderos), que permiten un acercamiento hacia los procesos de automatización para el análisis del comportamiento de las variables a costos asequibles para el agricultor-productor. 
Un elemento nuevo de estudio en la Corporación Universitaria Minuto de Dios Uniminuto, es la agrónica, la cual se enmarca en una propuesta académica de investigación en una maestría, por lo que este trabajo contribuye al fortalecimiento de la línea de monitoreo y supervisión enmarcado en IoT.

Por consiguiente, este artículo se organiza con una breve descripción del contexto, justificación y objetivo de la investigación, la segunda parte describe el modelo de funcionamiento del hardware y el software, la tercera parte aborda el caso de estudio para finalizar en los resultados y conclusiones.

\section{EsTAdo DEL ARTE}

La diversificación de la economía, que tradicionalmente se sostenía en el petróleo, empieza a migrar a diferentes sectores como la agricultura, la cual es parte fundamental de la economía de varios países. Por lo que la implementación de invernaderos ha permitido el monitoreo y control de microclimas favorables para el crecimiento de las plantas, a través de la aplicación de nuevas tecnologías como: redes inalámbricas de sensores (WSN), actuadores, controladores e Internet de las cosas (IoT). el objetivo de la investigación (Touhami, 2020) es proponer un diseño basado en estas nuevas tecnologías.

Un análisis de fenómenos que afectan el cambio climático amenaza cada vez más la calidad y cantidad de alimentos en los métodos de producción tradicionales en todo el mundo. Se espera que la agricultura inteligente sea una contramedida efectiva, aplicando tecnologías como Internet de las cosas (IoT) a la producción agrícola, incluido el método hidropónico. El objetivo la investigación (Angeloni, 2020) fue presentar una revisión literaria desde el enfoque de alimentos sostenibles, para luego explicar los beneficios de un innovador "dispositivo de invernadero hidropónico automático".

El sistema de cultivo hidropónico es un sistema agrícola que utiliza agua directa como nutriente, en algunos casos, y en otros, el agua lleva nutriente sin utilizar el suelo como medio de siembra. Este sistema permite a los pequeños agricultores tener la oportunidad de desarrollar su producción de cultivos con menos capital. Una de las tecnologías que se pueden aplicar en un sistema de cultivo hidropónico es el sensor. En la investigación (Nasution, 2017), la tecnología que se aplicará es la tecnología de sensores inalámbricos aplicada en la vida humana para ayudar a obtener información de forma rápida y precisa. Los sensores que se utilizarán en este estudio son sensores de $\mathrm{pH}$, sensores de conductividad, sensores de temperatura y humedad.

La investigación (Keerthana, 2018) presenta Hydroponic inteligente para el cuidado de plantas que usa una herramienta de administración de Internet de las cosas (IOT) llamada IOT talk. El modelo desarrollado incluye sensores, iluminación LED, rocío de agua y bomba de agua que pueden reducir efectivamente la concentración de $\mathrm{CO} 2$, la temperatura y aumentar el nivel del agua, respectivamente.

\section{Metodología}

Para el desarrollo de la investigación, se trabajó bajo una metodología con cinco pasos, el primero consistió en determinar los requerimientos del hardware y del software bajo las necesidades propias, el segundo paso definió las variables físicas de medición y los sensores, en el tercer paso se diseñó y construyó el prototipo experimental, el cuarto paso consistió en las pruebas simuladas y experimentales y el último paso fue el análisis de las variables; la Figura 1 describe la secuencia de la metodología.

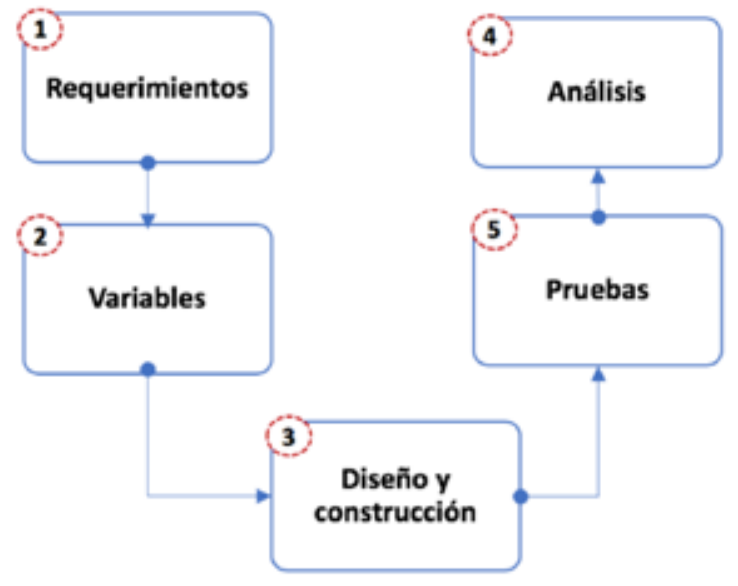

Fig. 1 Modelo de la metodología 


\section{MODELO}

El modelo se desarrolló e implementó en varias fases, las cuales se describen a continuación: a partir de la metodología se probaron diferentes tipos de microcontroladores y se compararon con Arduino; para la transmisión de datos a internet se usó el módulo de comunicaciones ESP8266. Para la trasmisión por Bluetooth ${ }^{\circledR}$, se trabajó el módulo $\mathrm{HC} 05$ con la finalidad de contar con una conexión a teléfono celular, los sensores usados fueron: ds18b20 y dht11, el modelo de hardware y el proceso general de comunicación se observan en la Figura 2.

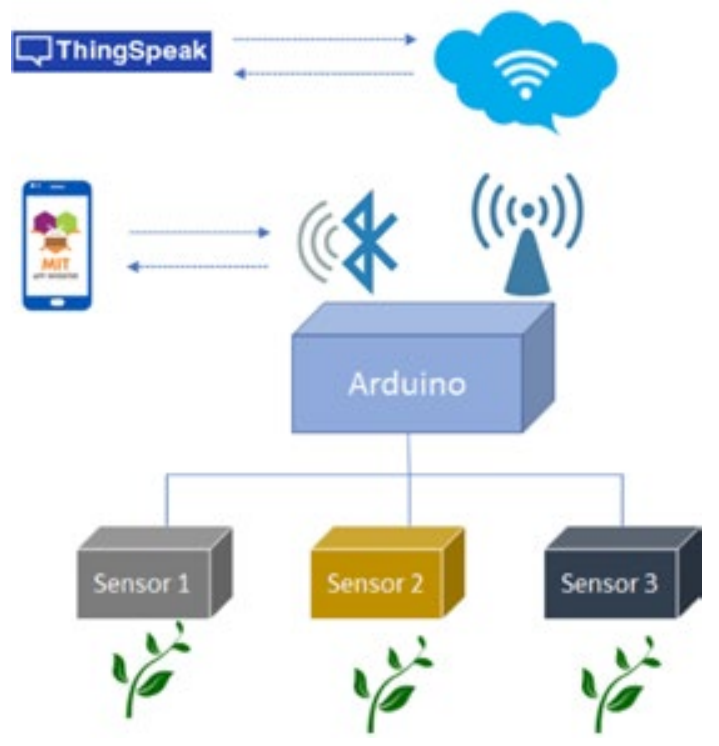

Fig. 2 Proceso del modelo del sistema de monitoreo

En la Figura 3 se observa el respectivo prototipo de diseño y se distingue una de las pruebas realizadas al tipo de plantas seleccionadas.

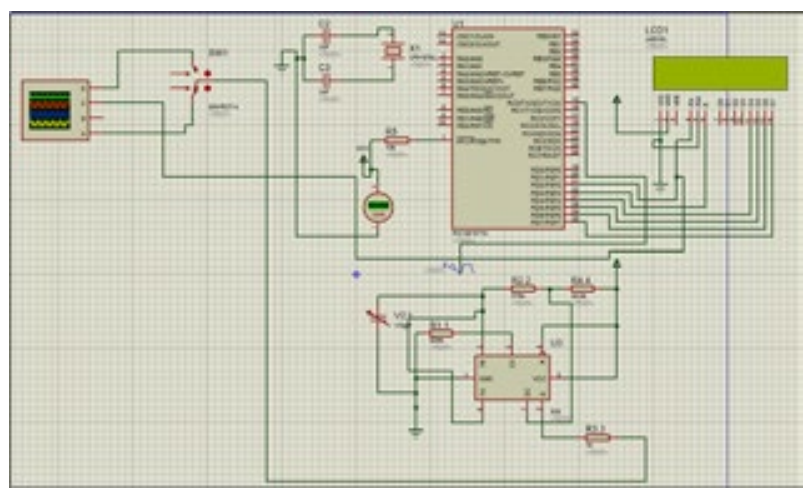

Fig. 3 Diseńo de prototipos
La estructura de la programación del prototipo de hardware se observa en la Figura 4 donde se hace énfasis en el sistema de comunicaciones WIFI, el cual se hace entre la plataforma Arduino y el módulo de comunicaciones ESP 8266 con comandos AT para la comunicación con la plataforma de IoT ThingSpeak ${ }^{\mathrm{TM}}$.

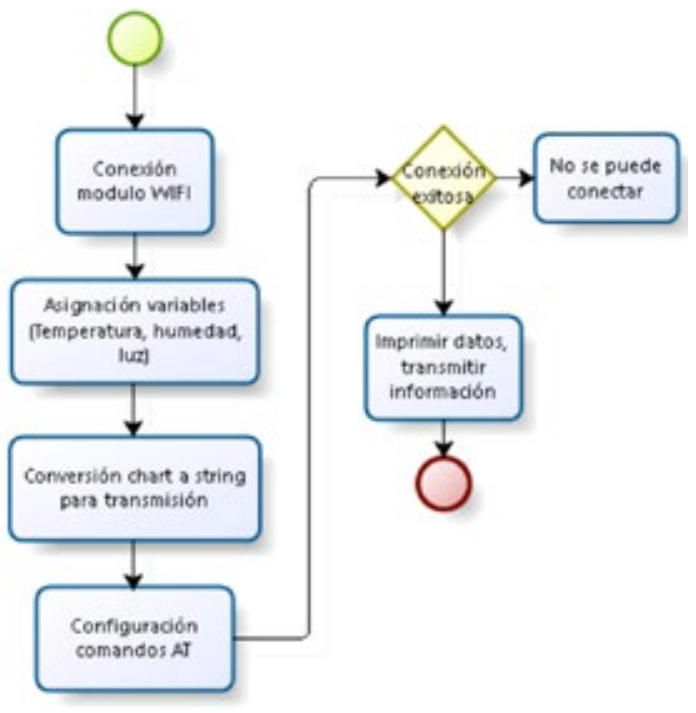

Fig. 4 Modelo de funcionamiento prototipo de hardware

El sistema de monitoreo también se implementó con transmisión vía Bluetooth ${ }^{\circledR}$, el cual se observa de manera clara en la Figura 5.

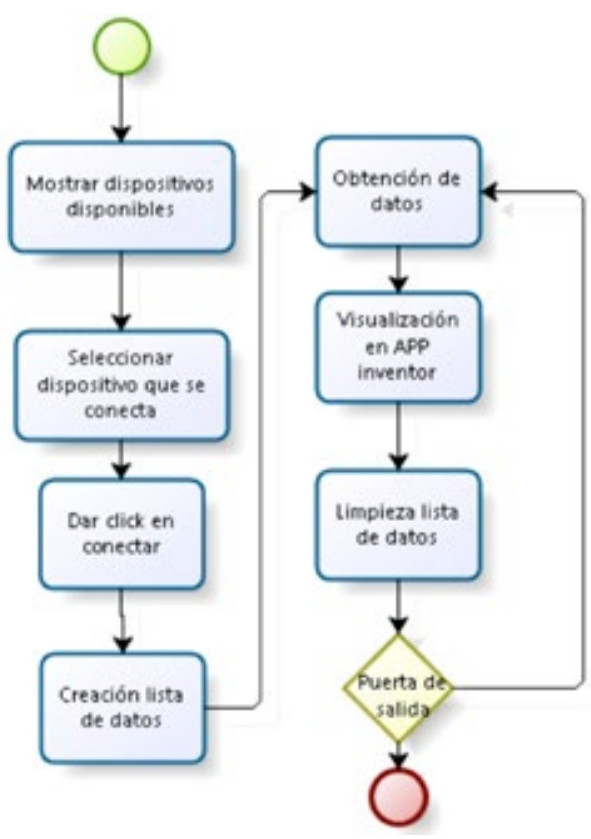

Fig. 5 Modelo de funcionamiento prototipo de APP 


\section{Resultados}

Para la verificación de los resultados se plantearon diferentes escenarios de pruebas ambientales, los anteriores se realizaron en espacios abiertos y cerrados, para garantizar la protección de los dispositivos; las pruebas se realizaron en interiores. La red de monitoreo se implementó en un cultivo de plantas ornamentales, para supervisar las siguientes variables agroecológicas: temperatura, luminosidad y humedad, como se puede ver en la Figura 6.

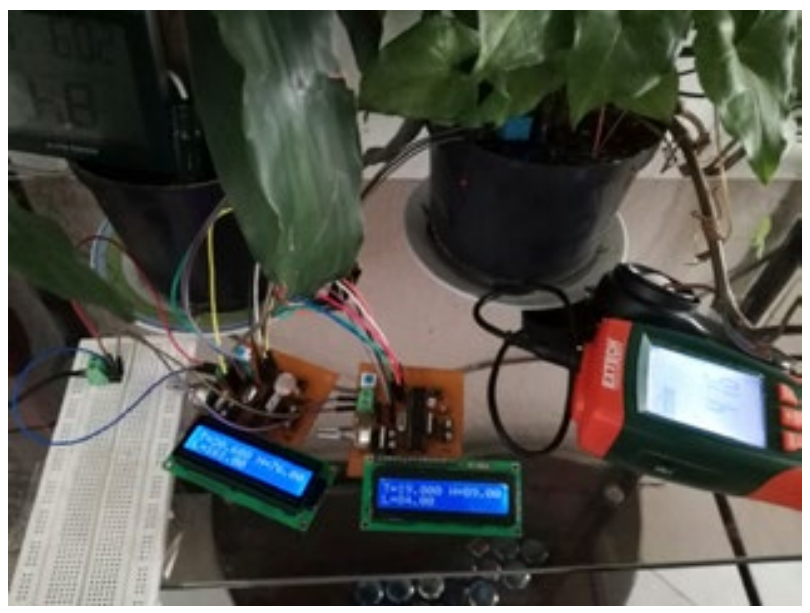

Fig. 6 Prueba básica de medición de variables

Para verificar los resultados se construyeron dos prototipos con los mismos sensores, permitiendo así la comparación de las variables, en el proceso previo experimental se calibraron a través de la comparación con patrones de laboratorio de las muestras.

La visualización de la información se realizó de tres formas, la primera fue local en el dispositivo, la segunda fue de forma inalámbrica a través de Bluetooth ${ }^{\circledR}$ para observar los datos en un celular y la tercera fue en cloud para cumplir el requerimiento de IoT en una plataforma de Matlab que es ThingSpeak ${ }^{\mathrm{TM}}$.

La configuración en la plataforma ThingSpeak ${ }^{\mathrm{TM}}$ usó tres canales para el monitoreo con las llaves públicas y los key, la prueba inicial se simuló en el software Proteus, construyendo los respectivos circuitos electrónicos, la Figura 7 enseña los canales utilizados en la plataforma de internet para dos variables, es importante resaltar que las plataformas web requieren de un tiempo de espera para la recepción de datos.

\section{My Channels}

\begin{tabular}{|c|c|c|c|c|c|}
\hline \multicolumn{5}{|l|}{ Name } & Created \\
\hline \multicolumn{5}{|c|}{ - TEMPERATURA } & \multirow[t]{2}{*}{$201704-03$} \\
\hline Phas & Pholic & seonos: & AAx"M & Dea mon/tepen & \\
\hline \multicolumn{5}{|c|}{ AUMEDAD } & \multirow[t]{2}{*}{20174409} \\
\hline Dhato & Dhaic & seono: & APakes & Des import / Expont & \\
\hline \multicolumn{5}{|c|}{ a INVERNADERO } & \multirow[t]{2}{*}{20174405} \\
\hline Anato & Proik & serong & APaken & Dea inport / Evoot & \\
\hline \multicolumn{5}{|c|}{ - INVERNADERO2 } & \multirow[t]{2}{*}{20170418} \\
\hline Shas & Pable & seonger & AAxess & Sta mpot / Espon & \\
\hline
\end{tabular}

Fig. 7 Canales usados para el monitoreo

La aplicación móvil se realizó con la App Inventor para realizar la conexión con Bluetooth ${ }^{\circledR}$, la Figura 8 muestra la implementación:

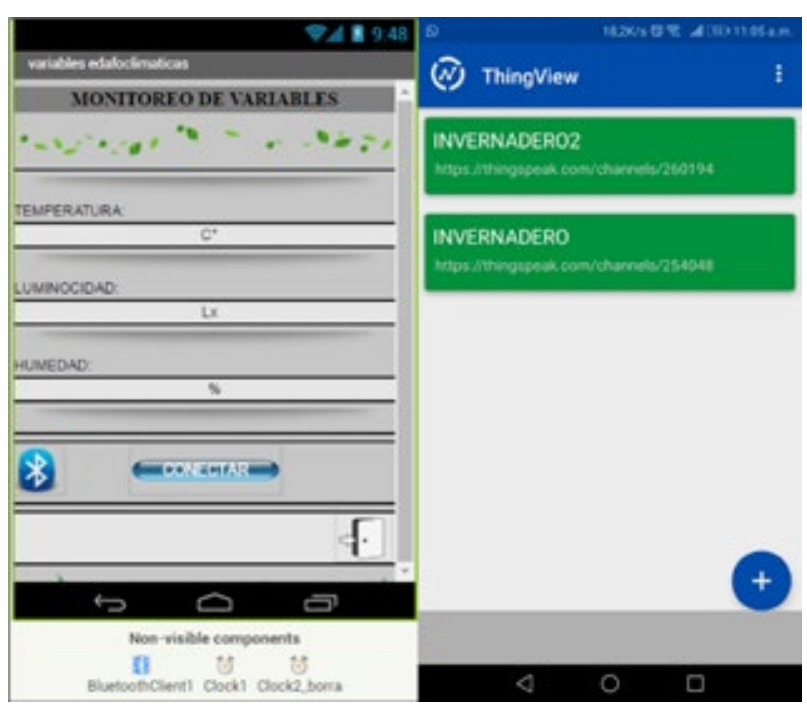

Fig. 8 App implementado

\subsection{Variable de temperatura}

La temperatura afecta la tasa de desarrollo de la planta, a través de sus distintas fases y la producción de hojas, tallos y otros componentes. Todos los procesos fisiológicos de la planta ocurren más rápidamente a medida que la temperatura aumenta, entre una temperatura base y una temperatura óptima (FAO, 2018). 
Dentro de los resultados obtenidos se destaca el monitoreo de esta variable en tiempo real, con una trasmisión de datos de forma directa al usuario. Lo anterior, con la finalidad de que los productores logren tomar decisiones acertadas y logren sortear, con mayor éxito, los posibles impases generados por elevadas temperaturas, o en su defecto, lo asociado a las bajas temperaturas, fenómeno conocido como heladas. La Figura 9 evidencia el registro de temperaturas de las plantas, monitoreadas con dos prototipos diferentes. Se puede observar la diferencia entre los dos con un valor de 0,7; el prototipo A realiza la transmisión de datos y se puede observar la diferencia de valores en el mismo tiempo, esto se debe a que la representación de los valores se ve retrasada por los tiempos de servicio de los servidores.

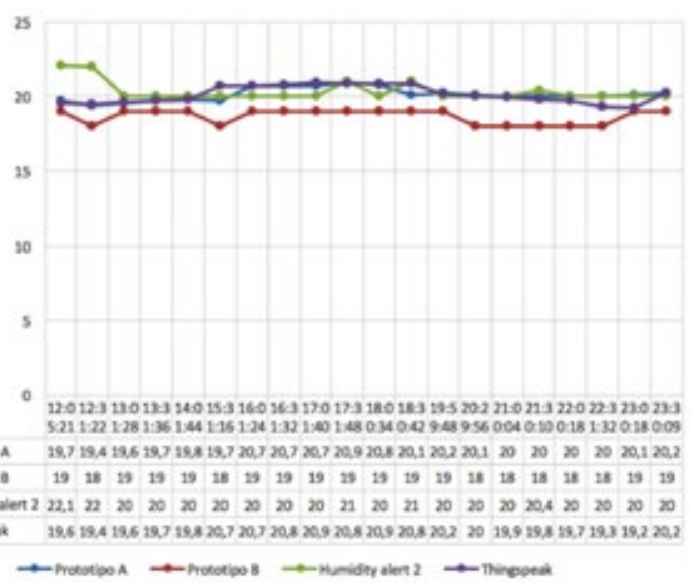

Fig. 9 Comportamiento de la temperatura de los dos prototipos

\subsection{Variable de humedad}

El contenido de humedad del suelo es un indicador complementario y necesario en numerosos análisis (Castellanos-Navarrete et al., 2013), frente a esta variable, se pudieron monitorear los respectivos comportamientos de la humedad del sustrato en relación con el agua aplicada en cada una de las macetas del estudio. Se evidenció un comportamiento similar y paralelo en cada uno de los prototipos diseñados frente al patrón establecido, lo que repercute de manera positiva en el conocimiento, por parte del agricultor, frente al recurso hídrico al interior del cultivo. Es de resaltar que "una correcta interpretación de las lecturas de humedad del suelo es muy importante para garantizar el manejo adecuado del riego y evitar excesos o en caso contrario déficits de agua que puedan afectar la planta (Zotarelli, Dukes \& Morgan, 2014). En la Figura 10 se puede evidenciar el comportamiento de esta variable.

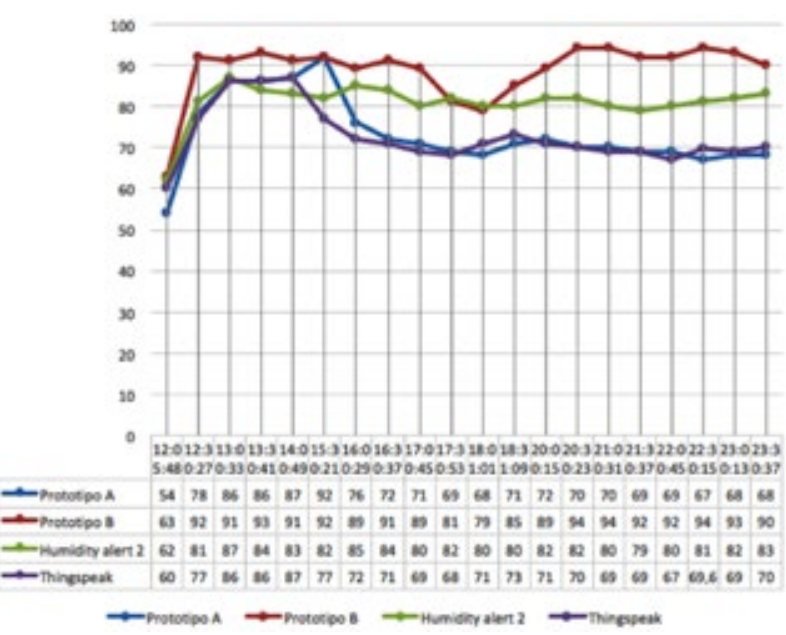

Fig. 10 Comportamiento de la humedad de los dos prototipos

\subsection{Variable de luminosidad}

Esta variable fue monitoreada de manera constante, arrojando como resultado diferentes comportamientos frente a la intensidad de luz recibida por las plantas. Es de resaltar que la luz influye tanto en el crecimiento (provisión de carbono en el proceso de fotosíntesis), como en el desarrollo de las plántulas (morfogénesis). El crecimiento de los sistemas productivos está influenciado por la cantidad de luz recibida durante todo el día (Adlercreutz, 2014). El monitoreo de esta variable permite en gran medida determinar, por parte del agricultor, si es necesario incrementar los niveles de esta variable por medio de luz artificial, que repercuta de manera positiva en el desarrollo vegetativo de los sistemas productivos, así como en la calidad de los productos cosechados frente a la acumulación de pigmentos fotosintéticos, lo que repercute en mejores coloraciones para el caso específico de flor de corte. La Figura 11 enseña los valores obtenidos frente a esta variable. 


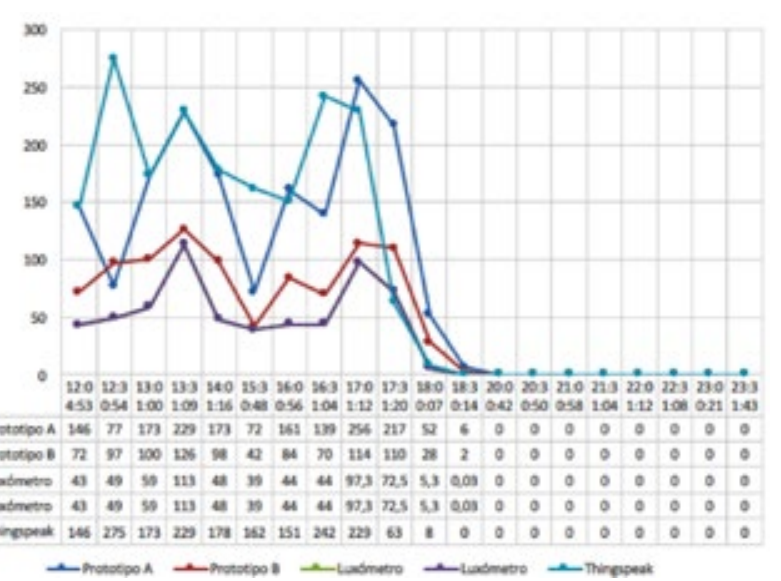

Fig. 11 Comportamiento de la luminosidad de los dos prototipos

En la Figura 12 se observan los resultados de la captura de datos en la plataforma ThingSpeak ${ }^{\mathrm{TM}}$ de las tres variables, en la Figura 13 se encuentran las gráficas de la aplicación desarrollada para móviles.

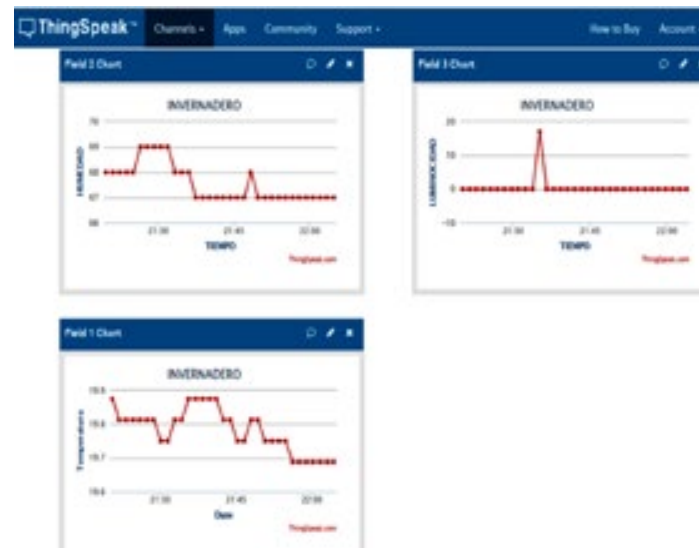

Fig. 12 Resultados en plataforma web

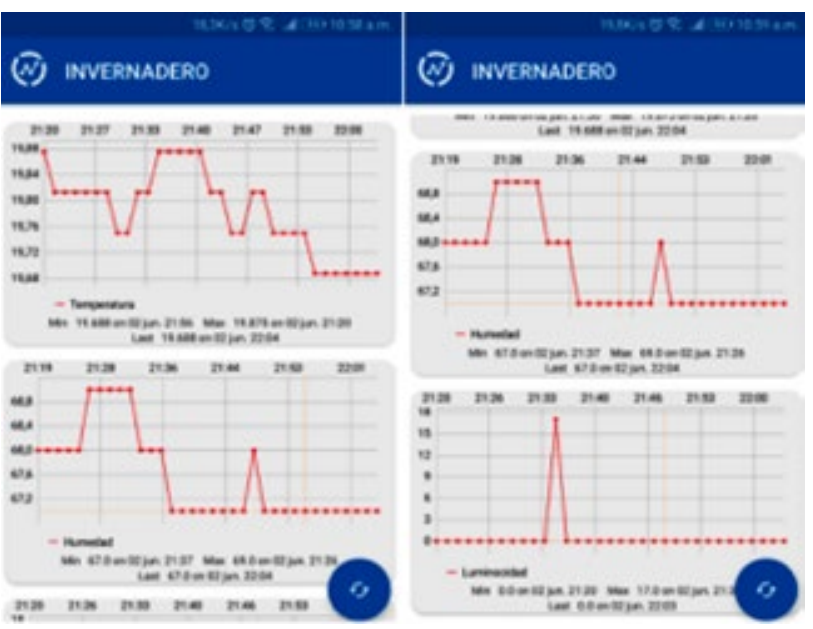

Fig. 13 Resultados en App.

\section{Conclusiones}

Realizar redes de sensores inalámbricos es de suma importancia, sí se pretende aumentar la competitividad en el sector agropecuario del país, y más, cuando se trabaja con tecnologías de bajo costo, que permite su replicabilidad para cultivos a pequeña escala y su aplicación por productores de bajos ingresos económicos. No obstante, es de resaltar, que los usos de los dispositivos deben ser seleccionados de forma adecuada, como es el caso puntual del sensor dht 11 , el cual tiene la debilidad de sulfatarse de forma rápida, lo que repercute en posibles errores en la medición de las variables.

Se logró determinar los valores de temperatura y humedad en un caso de estudio en particular; para los dos prototipos realizados se observó que existe un error, el cual depende de los sensores y de la resolución para la toma de datos. Para posteriores investigaciones se plantea determinar el error de los sensores de bajo costo parametrizados con patrones adecuados, así mismo sobre el tiempo de vida de los sensores para garantizar la correcta toma de datos.

Las mediciones pueden ser malinterpretadas según los tiempos de muestra, debido a que en la fuente no se toma la hora y no se transmite; el valor visualizado es el de la plataforma, lo que en casos puede generar confusión en la lectura.

Plantear el uso de tecnología para el sector agrícola y pecuario permite disminuir la brecha entre el agricultor-productor y las TIC, generando sinergias positivas que favorecen de manera directa el eslabón primario de la economía del país.

\section{REFERENCIAS}

Acuña Caita, J. F. (2009). Control climático en invernaderos. Bogotá: Universidad Nacional de Colombia.

Adlercreutz, E., Huarte, D., López, A., Manzo, E., Szczesny, A. \& Viglianchino, L. (2014). Producción hortícola bajo cubierta. Buenos Aires: Inta. Recuperado de: https://inta.gob.ar/sites/ default/files/script-tmp-inta-_prod_hort_bc.pdf 
Aguado da Costa, J. (2012). Desarrollo de un sistema automatizado para un invernadero. Tesis de grado. Valladolid: Universidad de Valladolid.

Alpi, A. \& Tognoni, F. (1999). Cultivo en Invernadero. Madrid: Mundiprensa.

Carrillo, D. \& Vázquez, J. (2008). Automatización de un invernadero con el plc s7-200. Tesis de grado. Zacatecas: Universidad Autónoma de Zacatecas.

Castellanos-Navarrete, A., Chocobar-Guerra, A., Cox, R., Fonteyne, S. Govaerts, B., Jespers, N., Kienle, F., Sayre, K. \& Verhulst, N. (2013). Contenido de humedad del suelo: guía útil para comparar las prácticas de manejo de cultivo. México: Centro Internacional de Mejoramiento de Maíz y Trigo CIMMYT.

Daniel Andrés Vega Castro es M.Sc en Educación. Docente e investigador de la Corporación Universitaria Minuto de Dios, Facultad de Ingeniería, Programa de Ingeniería Agroecológica.

Duván David Barbosa Pira es estudiante el programa de Tecnología en electrónica de la Corporación Universitaria Minuto de Dios (Uniminuto).

Edgar Aguirre Buenaventura es estudiante de doctorado en ingeniería de la Universidad Distrital Francisco José de Caldas, y trabaja como profesor en el programa de Tecnología en electrónica de la Corporación Universitaria Minuto de Dios. Sus líneas actuales de investigación son sistemas de alertas tempranas, sistemas multiagente, robótica y agrónica. eaguire@uniminuto.edu.

FAO (2018). Factores ambientales. Organización de las Naciones Unidas para la Agricultura y la Alimentación. Recuperado de: http://www.fao.org/docrep/006/x8234s/x8234s08.htm

Hassler Camilo Bueno Mesa es estudiante del programa de Tecnología en electrónica de la Corporación Universitaria Minuto de Dios (Uniminuto).

Juan Sebastián Sanabria Rodríguez es estudiante el programa de Tecnología en electrónica de la Corporación Universitaria Minuto de Dios (Uniminuto).
Montealegre, J. (2007). Modelo institucional del IDEAM sobre el efecto climático de los fenómenos El Niño y La Niña en Colombia. Bogotá: Instituto de Hidrología, Meteorología y Estudios Ambientales, Ideam.

Muñoz, J. \&. Nuñez, D. (2012). Automatización de invernadero en clima templado. Tesis de grado. Cali: Universidad San Buenaventura. Recuperado de: http://bibliotecadigital.usb.edu. co/bitstream/10819/865/1/Automatizaci\%C3\%B3n_Clima_ Templado_Ni\%C3\%B1ez_2012.pdf

Portafolio (2008). Invernaderos de alta tecnología en centros Corpoica. Recuperado de: http://www.portafolio.co/economia/finanzas/destinan-500-millones-construccion-invernaderos-altatecnologia-centros-corpoica-245138

Rodríguez, J. (2006). Diseño de un sistema inalámbrico para el monitoreo en tiempo real de temperatura y humedad relativa bajo invernadero. Tesis de grado. Bogotá: Universidad de la Salle. Recuperado de: http://repository.lasalle.edu. co/bitstream/handle/10185/16652/T44.06\%20R618d. pdf? sequence $=1$ \&isAllowed $=y$

Rodríguez Salgado, A. Ramírez García, L., Villanueva Bahena, O., Martínez, A., Bahena Pérez, A. (2011). Automatización de invernadero aplicando tecnología de punta, con fines de mejorar la producción agrícola a un bajo costo. México: Colegio Nacional de Educación Profesional Técnica Jiutepec.

Vega, D. (2011). Regulación agroecológica de poblaciones asociadas a cultivos. Bogotá: Corporación Universitaria Minuto de Dios / Instituto San Pablo Apóstol.

Zotarelli, L. Dukes, M. \&. Morgan, K. (2014). Interpretación del contenido de la humedad del suelo para determinar capacidad de campo y evitar riego excesivo en suelos arenosos utilizando sensores de humedad. The Institute of Food and Agricultural Sciences (IFAS), University of Florida. Recuperado de: https:// edis.ifas.ufl.edu/pdffiles/AE/AE49600.pdf 
Revista Especializada en Tecnología e Ingeniería 
